\title{
ARTÍCULOS ARBITRADOS \\ SEGREGACIÓN RESIDENCIAL SOCIOECONÓMICA \\ Y LA POLITICA PÚBLICA DE VIVIENDA SOCIAL. EL CASO DE LA CIUDAD DE CÓRDOBA (ARGENTINA)
}

Ana Laura Elorza

Licenciada en Trabajo Social y doctora en Ciencias Sociales (UNCuyo). Becaria posdoctoral CONICET, Instituto de Investigación de la Vivienda y Hábitat de la Facultad de Arquitectura, Urbanismo y Diseño. Docente de la Escuela de Trabajo Social, Universidad Nacional de Córdoba. analauraelorza@hotmail.com. 


\section{$\underline{\text { Resumen }}$}

En los últimos años el estudio de la segregación residencial se ha renovado, ya que se estaría profundizando, con un aumento de las consecuencias negativas en la calidad de vida material y simbólica de la población pobre segregada. La ciudad de Córdoba (Argentina) ha sostenido la estructura de desigual apropiación de los grupos sociales según su condición socioeconómica, en la que la mayoría de la población de bajos ingresos reside en las áreas periféricas con menores oportunidades para el desarrollo de actividades y de acceso a servicios y equipamientos sociales. Se analiza la incidencia de las políticas públicas de vivienda social en la dinámica del fenómeno de la segregación residencial socioeconómica en esta ciudad durante el período 2001-2008, con énfasis en la localización de los proyectos habitacionales en la periferia, la homogeneidad social de la población destinataria y la denominación estigmatizadora de esos nuevos barrios.

\section{Palabras clave}

Segregación residencial socioeconómica, política de vivienda social, pobreza.

\section{$\underline{\text { Abstract }}$ \\ Public Housing Policy and Socioeconomic Residential Segregation in the City Of Córdoba (Argentina)}

In recent years the study of residential segregation has been renewed, because segregation is increasing with a consequent increase of its negative consequences on the quality of material and symbolic life of the segregated poor. The city of Cordoba (Argentina) has maintained the structure of unequal appropriation of residential areas by social groups according to their socioeconomic status, in which most of the low-income population is living in outlying areas with fewer opportunities for the development of activities and access to social services and facilities. The impact of public policies on social housing in the dynamics of the phenomenon of socioeconomic residential segregation in this city is analyzed for the period 2001-2008, emphasizing the location of housing projects in the periphery, the social homogeneity of the target population and the stigmatizing designation of these new neighborhoods.

Keywords

Socioeconomic residential segregation, social housing policy, poverty. 


\section{INTRODUCCIÓN}

El tema de la segregación residencial ha sido abordado desde distintas perspectivas teóricas desde inicios de la Escuela de Chicago con los aportes de RoBert PARK. Sin embargo, en la década de los 90, a partir de la constatación de los impactos de la reestructuración del modelo de acumulación capitalista en las estructuras urbanas de las ciudades, este fenómeno toma una renovada relevancia; distintos investigadores destacan la profundización de la segregación residencial como un nuevo rasgo de las metrópolis (HARVEY, 2000; DAVIS, 2006; BORJA 2007, entre otros).

En América Latina, a pesar de que sus ciudades se caracterizaron desde su conformación por ser segregadas (JARAMILLO, 1999; SABATINI, 2003, 2004), a partir del proceso de reestructuración capitalista y la implementación de políticas de corte neoliberal, el análisis de este fenómeno se retoma, ya que estaría creciendo su escala geográfica y el aumento de las consecuencias negativas en la calidad de vida material y simbólica de la población pobre segregada (SABatini, CÁCERes y Cerda, 2001; Rodríguez y Sugranyes, 2005; Brain y SABATINI, 2006).

Al igual que la mayoría de las ciudades, la ciudad de Córdoba (Argentina) se ha caracterizado desde sus inicios por la desigual apropiación del espacio según la condición socioeconómica de los grupos sociales. A medida que la ciudad fue extendiéndose, en paralelo con el crecimiento poblacional, la configuración de nuevas formas de estratificación social y el valor del suelo urbano, se complejiza el fenómeno de segregación residencial socioeconómico (SRS); sin embargo, se ha sostenido la tendencia de que la mayoría de la población de bajos ingresos resida en las áreas periféricas, históricamente conformadas con menor oferta urbana, que se traduce en menores oportunidades para el desarrollo de actividades (laborales, educativas, culturales, etc.) y de acceso a servicios y equipamientos sociales.

Resultados de un estudio reciente nos permiten identificar la dinámica que ha tenido la SRS en esta ciudad durante los años 2001-2008, cuando se ha profundizado la concentración de los grupos de altos ingresos en una zona específica de la ciudad (en el sector del área central y del eje noroeste), mientras que los grupos de bajos ingresos se localizaron en la zona periférica de la ciudad (MARENGo y ElORZA, 2014, Elorza, 2014). En este artículo nos interesa identificar la incidencia de las políticas públicas de vivienda social en el desarrollo de este fenómeno durante ese período, dado que se ha desarrollado una fuerte política de vivienda social, el programa Mi casa, Mi Vida, en el marco del cual que se han 
1. Hace referencia a asentamientos urbanos que se configuraron a través de ocupaciones individuales (familiares) de tierras fiscales o privadas. En el contexto de surgimiento, el término "emergencia" hacía referencia a una residencia transitoria para las familias pobres hasta que pudieran acceder a un trabajo con salario justo y a una vivienda adecuada. Se convirtió en un "eufemismo cuando su carácter de emergencia, es decir, transitoria, se transformó en definitiva y dio paso a la designación popular de villa miseria" (ZILосCHI, 2007: 73). producido erradicaciones de setenta villas de emergencias ${ }^{1}$ y traslados a treinta y nueve nuevos barrios, en su mayoría localizados en la periferia de la ciudad.

Desde los ámbitos académicos y las organizaciones sociales se advierte sobre la profundización de la segregación residencial de la población de las villas. La hipótesis que sostenemos es que los proyectos de vivienda social implementados a través de políticas de vivienda social han incidido en la intensificación de la SRS y refuerzan las condiciones de reproducción de la pobreza de la población destinataria.

En este sentido, para el análisis de las vinculaciones entre la dinámica de la SRS y la política pública de vivienda social, este trabajo se estructura de la siguiente manera: primero, desarrollamos la conceptualización de la categoría conceptual de la SRS y la identificación de los factores que contribuyen a su producción y reproducción; segundo, presentamos una exploración de los antecedentes de estudios que dan cuenta de la incidencia de las políticas públicas de vivienda social y la SRS, haciendo hincapié en los aportes de académicos de la región latinoamericana. En tercer lugar, realizamos una descripción de la metodología empleada y el análisis de la dinámica que ha tenido la SRS en la ciudad de Córdoba durante el período estudiado. En cuarto lugar, indagamos sobre la modalidad de tratamiento del problema habitacional de los sectores de bajos ingresos desde las políticas públicas de vivienda social, en especial, la ejecución del Programa Mi Casa, Mi Vida implementado por el gobierno provincial y la incidencia de esta operatoria en la producción y dinámica de la SRS. Por último, presentamos una breve síntesis de los aspectos abordados en el trabajo y las conclusiones a las que arribamos.

\section{CONCEPTUALIZACIÓN DE LA SEGREGACIÓN RESIDENCIAL Y LOS FACTO- RES QUE CONFIGURAN SU PRODUCCIÓN}

SABATINI (2004) entiende a la segregación residencial como la aglomeración en el espacio de familias de una misma condición social, más allá de cómo se definan las diferencias sociales. Por lo tanto, consiste en una relación espacial: de proximidad territorial o de separación entre personas pertenecientes a un mismo grupo social.

A diferencia de los estudios sobre la segregación socioterritorial en Europa y Estados Unidos que han estado vinculados con la segregación residencial racial y étnica (MASSEY y Denton, 1988; Bourdieu, 1999; WaCQuant, 2001; 2007; ARBACI, 2008), la preocupación 
por el análisis de este fenómeno según la condición socioeconómica de la población es prioritariamente de nuestra región (RodRíGUez Vignoli, 2001; SABATINI, 2003). Esta diferencia de analítica se puede vincular con las determinaciones histórico-sociales que han ido confluyendo en la producción de las ciudades de los distintos continentes.

La segregación residencial presenta tres dimensiones para su examen: 1) el grado de concentración espacial de los grupos sociales (en términos de localización urbana), 2) la homogeneidad que presentan las diferentes áreas de la ciudad en términos de su composición social y 3) la percepción que las personas tienen de la segregación, como fenómeno espacial. SABATINI (2004) explica que las dos primeras dimensiones hacen referencia al aspecto objetivo de la segregación, aunque cada una capta una arista distinta del fenómeno, mientras que la tercera se relaciona con aspectos subjetivos. En el presente trabajo, si bien se reconoce la importancia de esta última dimensión, enfocamos el análisis en la dimensión objetiva, vinculada con la manifestación de la segregación en el territorio.

RodRíGuez Vignoli (2001) refiere que en las dos primeras dimensiones hay una cierta superposición, ya que la localización de un grupo determinado en una cierta área implica la configuración de un área homogénea. Sin embargo, la distinción de estas dos dimensiones tiene sentido. La primera tiene lugar cuando algún grupo social registra un sesgo residencial global, es decir, todos (o la gran mayoría) de sus miembros se localizan en una zona determinada del territorio, sin importar si en dicha zona hay otros grupos sociales. Esta segregación por localización de grupo opera cuando, en una situación en la que hay varios grupos sociales, uno o más no están dispersos por el territorio, sino que se encuentran concentrados en una zona específica. En cambio, la segunda dimensión, que puede denominarse por exclusión, atañe a la ausencia de mezcla o integración de grupos sociales en espacios comunes. En esta dimensión de la segregación, un grupo social no se mezcla con el resto aunque esté diseminado en varias partes de la ciudad. Así se originan zonas homogéneas en un contexto heterogéneo, lo que probablemente dificulta la interacción con otros grupos sociales.

SARAVI (2008) enriquece el análisis aclarando que cuando hablamos de la segregación en términos de la distribución de la población en el espacio, del nivel de concentración de determinados grupos en ciertas áreas de la ciudad o del grado de homogeneidad social que presentan áreas específicas no se trata simplemente de un criterio de diferenciación, sino al mismo tiempo de un criterio clave de jerarquización y, más específicamente, de desigualdad social. 
Más allá de estas definiciones conceptuales, para poder comprender este fenómeno debemos remitirnos a los factores que intervienen en su generación y los procesos producidos en los últimos años que estarían dando cuenta de una profundización de la segregación en las ciudades, en especial, en América Latina. JARAMILLO (1999) sostiene que las diferencias entre los lugares de residencia de los grupos de altos ingresos y los otros grupos sociales eran abismales, y esa alteridad también se expresaba en separación espacial, como lo atestiguan los mencionados cinturones de la miseria, barriadas y barrios bajos; pero hasta cierto punto se compartía el uso del espacio central en la ciudad y había una localización de los diferentes grupos relativamente contigua.

Sin embargo, en las últimas dos décadas, con el crecimiento de las ciudades, los cambios sociales traducidos en nuevas preferencias residenciales de los grupos de altos ingresos, el aumento de los precios de la tierra y las dificultades de acceso a suelo urbano por parte de los grupos de menores ingresos podría decirse que la segregación se ha agudizado. Este autor destaca las diferencias de la manifestación de este fenómeno en las ciudades de la región en comparación con las ciudades estadounidenses (donde se habla del modelo "rosquilla") y las europeas.

En las metrópolis latinoamericanas la separación entre los grupos de diversos ingresos es más acentuada; los grupos más ricos siguen un comportamiento de autosegregación en sus funciones residenciales con rasgos particulares: las islas de opulencia siguen una pauta de periferización que generalmente sigue una sola dirección (cono de altos ingresos), y el nodo de estos asentamientos se desplaza hacia la periferia en una fuga continua hacia sitios cada vez más alejados, con una acentuada dicotomización socioespacial como resultado (JARAMILLO, 1999).

En este proceso, el rol del mercado de suelo y de la acción estatal es central. Por un lado, el mecanismo de los precios del suelo juega un papel decisivo en la segmentación social del espacio, ya que garantiza el acceso exclusivo de grupos privilegiados a territorios positivamente connotados con cualidades paisajísticas de interés, por lo general promovidos desde las estrategias de marketing de los desarrollistas inmobiliarios.

Por otro lado, el Estado interviene en la producción de la segregación residencial socioeconómica, ya sea de manera directa, a través de la localización de la población de bajos ingresos en áreas periféricas de la ciudad por medio de las políticas de vivienda, o de manera indirecta, por su injerencia en la definición del precio del suelo urbano, a través 
de la reglamentación urbana de uso de suelo y la modalidad de edificación, la producción de obra pública, el desarrollo de políticas habitacionales (JARAMILLO, 1999; SABATINI, 2003; HidALgo DatTWyler, 2007).

No obstante, muchas veces estos condicionantes (el precio de la tierra y las normativas urbanas) no son suficientes para sostener el proceso de segregación de los grupos de altos ingresos, lo cual ha conducido a su reforzamiento mediante instrumentos más explícitos: la clausura física de sus espacios, a través de productos residenciales cerrados como los countries, condominios de torres en altura, etc. (JARAMILLO, 1999: 120). CALDEIRA (2010) expone que la opción de la elite de substituir los espacios públicos y urbanos tradicionales por nuevos espacios privatizados es justificada por el temor a la violencia delictiva. La privatización y las fronteras rígidas (materiales o simbólicas) que fragmentan el espacio sirven para mantener separados los grupos; estas separaciones se van creando de diferente forma (con muros, con dispositivos de diseño, la desconfianza, el miedo a la delincuencia), tienen como consecuencia la discriminación y criminalización de los más desfavorecidos y contribuyen de esta manera a la producción de los estigmas territoriales.

\section{LAS POLÍTICAS PÚBLICAS DE VIVIENDA SOCIAL Y LA SRS}

Es rica y diversa la producción de investigaciones referidas a la relación entre política pública de vivienda social y la producción de la segregación residencial por la condición socioeconómica de la población en las ciudades de América Latina. Si bien históricamente las políticas de vivienda social han sido criticadas por la localización de los proyectos habitacionales en áreas periféricas y con limitaciones en el acceso a los servicios públicos y equipamientos sociales y culturales (Yujnovski, 1984; Pradilla Cobos, 1987), en las últimas décadas, a partir de las transformaciones ocurridas en las ciudades como consecuencia de los cambios en el régimen de acumulación capitalista y la orientación de las políticas habitacionales, estos problemas se han reforzado.

Por la prevalencia de la lógica del mercado en las políticas de vivienda operada por las grandes empresas del negocio inmobiliario, en el que prima el objetivo de mayores ganancias, la localización de los proyectos habitacionales está cada vez más alejada de los sectores de centralidad, con superficies mínimas habitables, peor calidad constructiva de las viviendas, conjuntos de gran tamaño y mayores estigmas por ser residentes de barrios de vivienda social. 
2. El autor menciona que el déficit disminuyó de 888.681 unidades en 1992 a 450.000 en el año 2000.
Hidalgo Dattwyler (2007) plantea que lo novedoso de la política de vivienda desarrollada en las últimas décadas en Chile es que los nuevos proyectos habitacionales ya no se encuentran en la periferia de la ciudad motorizando la expansión urbana, sino que estos conjuntos se levantan en espacios distantes de la mancha urbana, tanto en el medio rural como en las ciudades menores existentes en los confines de las grandes conurbaciones. Esta política, si bien ha tenido como resultado una importante disminución del déficit habitacional ${ }^{2}$, ha traído aparejada una serie de externalidades en las ciudades chilenas, que se manifiestan en procesos de segregación social y fragmentación física del espacio urbano (Rodríguez y Sugranyes, 2005; Brain y Sabatini, 2006).

En México, la política de vivienda social producida desde los años 90 también se ha caracterizado por la activa participación del sector inmobiliario privado; su intervención no fue solo en la construcción de viviendas, sino también en la adquisición del suelo, su urbanización, la construcción de viviendas, la promoción e, incluso, los mecanismos de financiamiento, en los que se instauró un sistema predominantemente privatizado y mercantilizado (Esquivel, Maya y Cervantes, 2005). Esto ha tenido como correlato la construcción de megaproyectos residenciales de gran superficie y elevado número de viviendas (Rodríguez Chumillas, 2006), emplazados en municipios distantes de las grandes ciudades del país, sin una adecuada previsión para la dotación de servicios urbanos y comunitarios, ya que los promotores inmobiliarios se limitan a proporcionar solo los elementos mínimos indispensables para cumplir con la normativa establecida.

En Argentina, un informe del Programa de Naciones Unidas por el Desarrollo (PNUD) (2009) analiza el fenómeno de la segregación residencial socioeconómica y plantea los límites de las políticas habitacionales desarrolladas en los últimos quince años, ya que los distintos programas no inciden en la composición social de los barrios, que siguen habitados por población con alto grado de vulnerabilidad, lo cual trae aparejadas condiciones de reproducción de la pobreza.

En el ámbito de la ciudad de Córdoba, distintos estudios sobre las políticas de vivienda social de erradicación de villas desarrolladas durante las últimas tres décadas muestran que la nueva localización de los conjuntos habitacionales dirigidos a la población de bajos ingresos se ha realizado en la periferia, atendiendo a una oferta de parcelas para ser urbanizadas de poco valor, una oferta accesible, con un bajo costo (MARENGO, 2004; TECCO Y FernándeZ, 2005; MARENGO y ElorZa, 2009, 2014). 
En resumen, podemos decir que en los estudios realizados en diferentes ciudades de la región se identifican ciertos rasgos constantes de las políticas de vivienda social, pero profundizados por el rol del Estado como garante de la supremacía del mercado del suelo e inmobiliario, en las que cada vez más la localización de los proyectos de vivienda es periférica, lo que ha devenido en profundos problemas, como el aumento de las distancias respecto de los lugares de servicio, la desestructuración de las redes sociales primarias y secundarias como consecuencia de la inadecuada localización en los límites de la gran ciudad y la construcción de territorios socialmente homogéneos, lo que reduce las posibilidades de interacción entre grupos sociales y dificulta la reproducción social de los sectores de bajos ingresos.

\section{LA SRS EN LA CIUDAD DE CÓRDOBA}

Desde su conformación, la ciudad de Córdoba se ha caracterizado por ser segregada, primero, marcando diferencias entre el espacio central - territorio de los colonizadores españoles - y la periferia - territorio de los pueblos originarios y de las actividades de servicios - A medida que la ciudad fue extendiéndose, en paralelo con el crecimiento poblacional, la configuración de nuevas formas de estratificación social y el valor del suelo urbano, se complejiza el fenómeno de segregación residencial socioeconómico.

Para el estudio de la dinámica de la SRS durante el período 2001-2008, aplicamos una metodología cuantitativa; el análisis realizado es a escala de fracciones censales, lo que nos posibilitó identificar el comportamiento de las dimensiones objetivas de la segregación: la concentración espacial de los grupos sociales y la homogeneidad que presentan las diferentes áreas de la ciudad en términos de su composición social. Debido a que los censos nacionales de población y vivienda en Argentina no incorporan indicadores de medición de la segregación, se tomaron los años de estudio del jefe de hogar y las Necesidades Básicas Insatisfechas (NBI por persona) como variables de segmentación socioeconómica, lo cual permite extrapolar la pertenencia social de los hogares en diferentes grupos socioeconómicos ${ }^{3}$.

El análisis del nivel de homogeneidad-heterogeneidad en las distintas subdivisiones territoriales (fracciones censales) se realizó a partir de la media de las variables seleccionadas y el cálculo estadístico de la varianza y el coeficiente de variación, que expresa la magnitud de la desviación estándar en función de la media de la distribución (ARRIAGADA Y RODRíGUEZ, 2003). Para dicho cálculo se utilizaron datos secundarios: el Censo de Población Nacional
3. Duhau (2013) menciona que para estratificar a los individuos $u$ hogares, la mayor parte de los investigadores optan por recurrir a variables tales como ingreso, escolaridad, ocupación o bienes poseídos. La elección de la variable que establecemos se basa en el supuesto de que existe una correlación entre la educación del jefe de hogar y la probabilidad de obtener mayores ingresos familiares. 
4. Se tomó este período dado que a la fecha de desarrollo de la investigación no se contaba aún con los datos procesados del Censo Nacional de Población y Vivienda 2010. del año 2001 realizado por el Instituto Nacional de Estadísticas y Censos (Indec) y el Censo Provincial de Población 2008. ${ }^{4}$

El procedimiento de medición de la segregación se dividió en dos instancias, en relación con cada una de las dimensiones objetivas de la segregación. Para conocer la concentración espacial de los grupos sociales en sectores de la ciudad, se calculó la media de años de estudios de los jefes de hogar y el valor del coeficiente de variación de cada unidad geográfica (fracciones censales) respecto de aquella para los dos cortes temporales (2001 y 2008). Con los resultados obtenidos, se realizó una clasificación en cuatro intervalos, según la intensidad del coeficiente de variación de la variable analizada por fracción. Es decir, se consideraron, en un primer intervalo, aquellos valores de variación comprendidos entre el $1 \%$ y el $10 \%$ (por encima y por debajo de la media), lo cual señala heterogeneidad en la distribución y, consecuentemente, un bajo valor de segregación. El segundo intervalo agrupa los valores comprendidos entre el $11 \%$ y el 20 $\%$ (por encima y por debajo de la media), los cuales se consideran valores moderados de segregación. El tercer intervalo incluye valores comprendidos entre el $21 \%$ y el $30 \%$ (por encima y por debajo de la media), que se consideran valores altos de segregación. Finalmente, el cuarto intervalo abarca valores que superan el $30 \%$ (por encima y por debajo de la media), que se consideran de muy alta segregación. Estos intervalos han sido representados en planos de la ciudad (figura N. ${ }^{\circ} 1$ ) a los efectos de identificar los sectores urbanos en situación de mayor concentración en los diferentes cortes temporales y analizar su dinámica.

Los resultados de este procedimiento demuestran un sostenimiento del patrón tradicional de segregación de las ciudades latinoamericanas; se identifica la distribución de la población en determinadas áreas de la ciudad: el área central se encuentra ocupada por los sectores de altos ingresos y su localización residencial se expande hacia el sector noroeste de la periferia de la ciudad conformándose un "cono de alta renta" (SABATINI, 2003); en el área intermedia de la ciudad se evidencia la mayor presencia de los sectores medios y en el área de la periferia, con excepción del eje noroeste, predomina la población de bajos ingresos.

El análisis comparativo según esta variable entre los cortes temporales 2001-2008 permite observar un comportamiento contradictorio de este fenómeno. Por un lado, se han ampliado las áreas de baja y moderada segregación (áreas amarillas y naranjas), lo que significa un mayor esparcimiento de la localización de los sectores de ingresos medios en la ciudad; el proceso de localización de los grupos de bajos ingresos ha tenido una dinámica de 


\section{SRS en la ciudad de Córdoba segun Nivel de Años de Estudio de Jefe de Hogar}
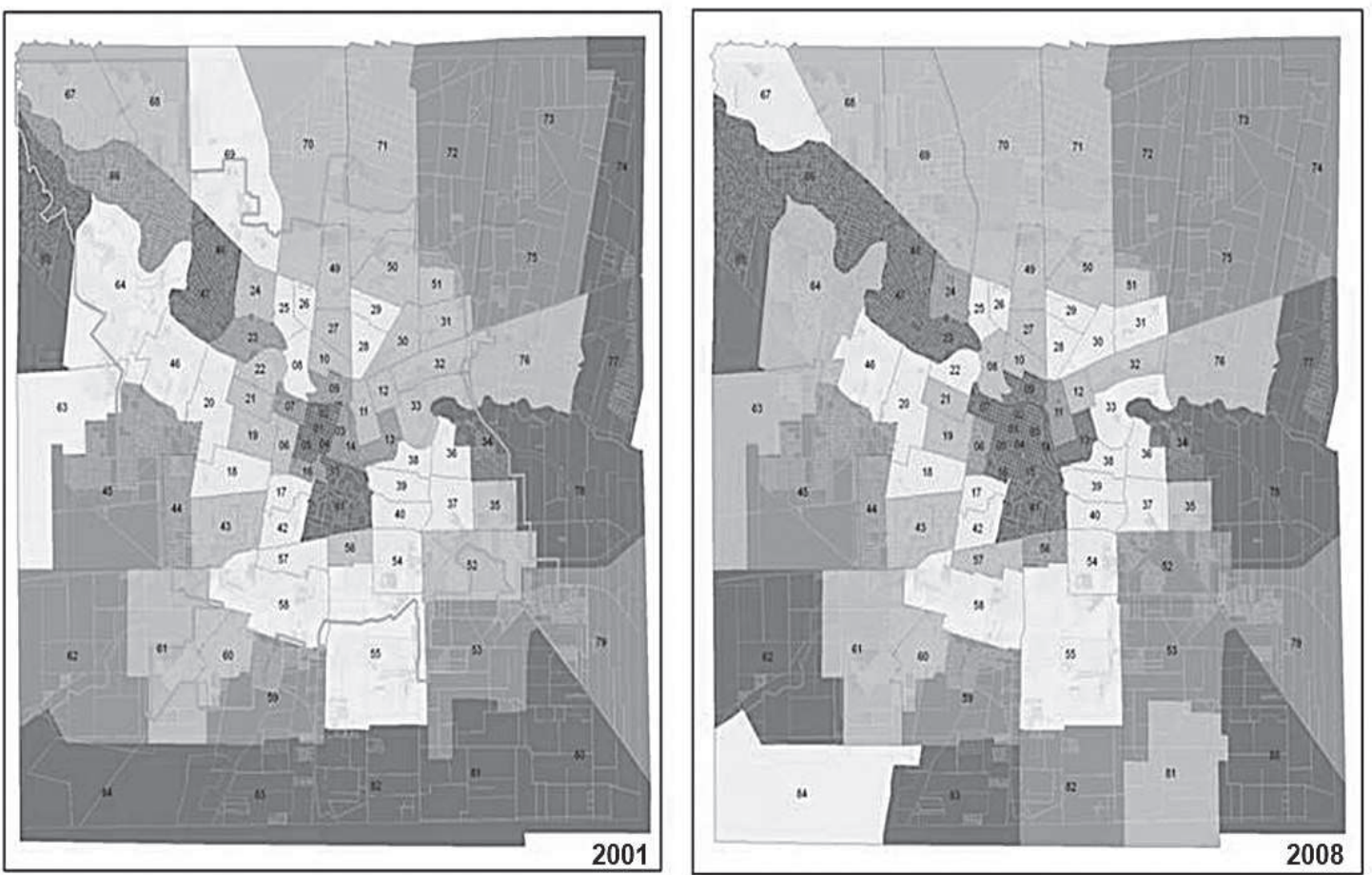

REFERENCIAS Desviación Estandar de la media

Variación del 1 al $10 \%$ - Nivel bajo de segregación

Variación del 11 al $20 \%$ (por encima de la media)- Nivel moderado de segregación

Variación del 11 al $20 \%$ (por debajo de la media)- Nivel moderado de segregación

Variación del 21 al $30 \%$ (por encima de la media)- Nivel alto de segregación

Variación del 21 al $30 \%$ (por debajo de la media)- Nivel alto de segregación

Variación mas del $30 \%$ (por encima de la media)- Nivel muy alto de segregación

Variación mas del $30 \%$ (por debajo de la media)- Nivel muy alto de segregación

$$
\begin{array}{r}
\text { Fuente: Censo Nacional de Poblacion } \\
\text { y Vivienda } 2001 \text { y Censo Provincial } \\
\text { de Poblacion y Vivienda } 2008
\end{array}
$$

Figura 1. Segregación Residencial Socioeconómica en la ciudad de Córdoba según años de estudio del jefe de hogar (2001-2008) 
dispersión en el sector periférico de la ciudad (áreas celestes y azules). Por otro lado, en sentido opuesto, se han consolidado los sectores de la ciudad identificados con alta y muy alta segregación de la población de más alto ingreso, que se concentra en el área central y noroeste de la ciudad (áreas rojas).

A partir de estos resultados, avanzamos en la medición de la segunda dimensión objetiva de la segregación, la homogeneidad social que presentan los sectores de las ciudades (áreas de la ciudad en términos de su composición social). En particular, nos ha interesado identificar los sectores de la ciudad donde hay una concentración de población en condiciones de pobreza estructural, buscando determinar una de las aristas del fenómeno de la SRS, vinculada con el proceso de aislamiento de los pobres en determinadas áreas de la ciudad.

El procedimiento que realizamos consistió en el cálculo de la media de población con NBI para la ciudad, a partir de la cual identificamos solo las fracciones en que la cantidad de población con NBI supera esa media. Cabe aclarar que solo en 29 fracciones sobre un total de 84 la población con NBI es superior a la media de la ciudad, lo que da cuenta de la desigual distribución en la localización de los distintos grupos sociales en ella. A modo ilustrativo, ejemplificamos esto con el caso de fracciones del área central (fracciones $1 \mathrm{y}$ 4 ) y del sector noroeste (fracciones 48,65 ), donde se registra un porcentaje menor al $1 \%$ de población con NBI; en oposición, en las fracciones en el sector sur (83) este porcentaje aumenta al $33 \%$.

Con fines analíticos, para el caso de estas fracciones censales, como en la instancia anterior, hemos realizado una categoría de cuatro intervalos según la intensidad de desviación por fracción: nivel bajo de segregación (con variación del 1 al $10 \%$ ), nivel moderado de segregación (con variación del 11 al $20 \%$ ), nivel alto de segregación (con variación del 21 al $30 \%$ ) y nivel muy alto de segregación (con una variación superior al $30 \%$ ). Estos resultados también han sido espacializados en el plano de la ciudad y superpuestos con los resultados del análisis previo obtenido con la variable nivel de años de estudio de los jefes de hogar.

Como se puede observar en la figura N. ${ }^{\circ} 2$, con la superposición del análisis de las dos variables utilizadas hay una clara correspondencia: por un lado, entre las fracciones en las que la media de años de estudio de los jefes de hogar es inferior a la media y las fracciones con mayor cantidad de población con NBI (señaladas con círculos) y, por el otro, entre 


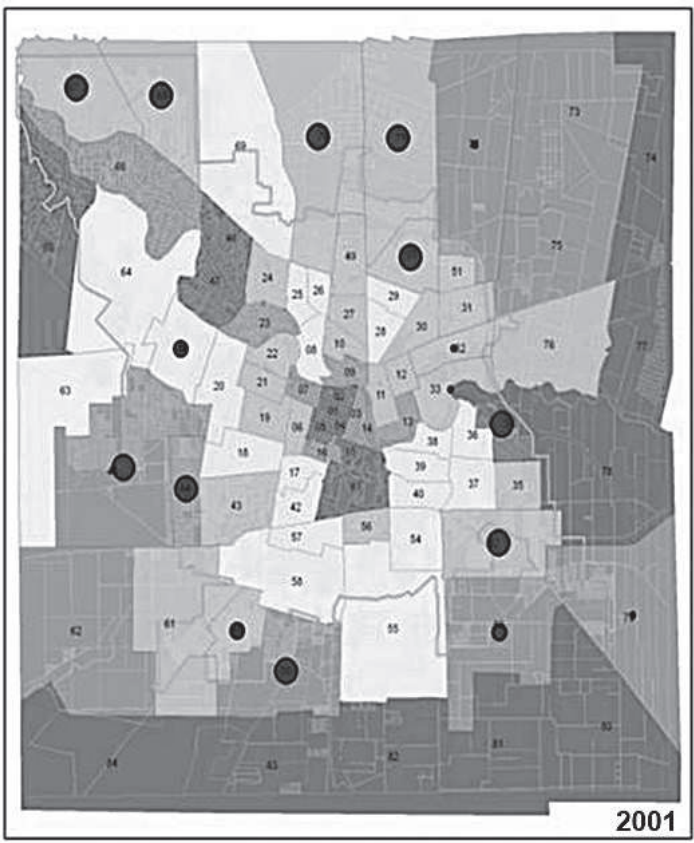

REFERENCIAS Desviación Estandar de la media

Variación del 1 al $10 \%$ - Nivel bajo de segregación

Variación del 11 al $20 \%$ (por encima de la media)- Nivel moderado de segregación Variación del 11 al $20 \%$ (por debajo de la media)- Nivel moderado de segregación

Variación del 21 al $30 \%$ (por encima de la media)- Nivel alto de segregación

Variación del 21 al $30 \%$ (por debajo de la media)- Nivel alto de segregación

Variación mas del $30 \%$ (por encima de la media)- Nivel muy alto de segregación

Variación mas del $30 \%$ (por debajo de la media)- Nivel muy alto de segregación

$$
\begin{array}{r}
\text { Fuente: Censo Nacional de Poblacion } \\
\text { y Vivienda } 2001 \text { y Censo Provincial } \\
\text { de Poblacion y Vivienda } 2008
\end{array}
$$

Figura 2. Segregación Residencial Socioeconómica en la ciudad de Córdoba según años de estudio de jefe de hogar y concentración de población con NBI (2001-2008) 
las fracciones que conforman el área central y del corredor noroeste, que históricamente ha sido el territorio residencial de los grupos de altos ingresos, en las que tiene menor presencia la población con NBI.

La comparación realizada (2001-2008) demuestra que la distribución de la población en situación de NBI mantiene una tendencia de localización en determinadas áreas periféricas de la ciudad. Mientras que en 2001, el 67,20 \% de esta población reside en la periferia de la ciudad, en 2008 este porcentaje desciende levemente al 65,80\%.

En resumen, durante el período analizado, en la ciudad de Córdoba se mantiene el patrón tradicional de segregación de las ciudades de América Latina, que consiste en una distribución de la población en determinadas áreas de la ciudad. Sin embargo, la dinámica que ha tenido la SRS puede analizarse como un doble proceso: por un lado, de mayor concentración de la población de altos ingresos en un área de la ciudad y, por el otro, de sostenimiento de la localización de los grupos de bajos ingresos en otras, lo cual representa situaciones problemáticas especialmente para el segundo grupo, ya que deben enfrentar en su cotidianidad problemas de accesibilidad y la carencia o la mala calidad de servicios y equipamientos en sus lugares de residencia y también los problemas derivados del aislamiento físico, como falta de oportunidades laborales, culturales, discriminación, etc., lo que contribuye a la producción y reproducción de las situaciones de pobreza.

\section{LA POLÍTICA PÚBLICA DE VIVIENDA SOCIAL EN LA CIUDAD DE CÓRDOBA (2001-2008)}

A partir de las transformaciones en el interior del modelo de acumulación y la implementación con mayor énfasis de las políticas neoliberales en la década del 90, en Argentina se produjeron modificaciones de las políticas sociales que se caracterizaron por el achicamiento del gasto social y la retirada del Estado de ámbitos públicos. Estos cambios estuvieron regidos por los principios del Consenso de Washington, delimitados por los organismos internacionales de financiamiento, y crearon una nueva manera de afrontar la cuestión social, caracterizada por un enfoque residual. Es decir, se transformó la política social fundamentada en los derechos sociales ciudadanos para dar paso a acciones de mitigación de la pobreza, fragmentadas y destinadas solo a los sectores definidos como pobres (Espina Pietro, 2008). 
En el plano sectorial de la política pública social, el enfoque de las políticas de vivienda se comienza a regir en términos de "facilitar" el funcionamiento de los mercados (de suelo y de la construcción) y de "aliviar" la pobreza, con un claro componente focalizador hacia la población definida como "pobres", sin acceso a una vivienda vía el mercado formal. En este marco, en la ciudad de Córdoba se desarrolla un programa de relocalización de villas denominado Mi Casa, Mi Vida, que tiene como objeto relocalizar a las familias residentes de villas ubicadas en la orilla del río Suquía. Por la importancia de su escala de intervención y de los impactos que ha tenido en la configuración de la ciudad y en el fenómeno de la segregación residencial socioeconómica, esta operatoria es analizada a continuación.

En el año 2001, el Poder Ejecutivo de la provincia de Córdoba declara la emergencia hídrica y la urgente relocalización de los grupos vulnerables afectados por las inundaciones debido al desborde del río Suquía. En este marco, la Dirección Provincial de Vivienda (DIPROVI) determina objetivos, requisitos y el paquete de normativas que abarcaron las acciones para el plan de rehabilitación habitacional de grupos vulnerables afectados por las inundaciones en la ciudad de Córdoba. Dicho plan se difundió públicamente a través de la propaganda oficial como el programa Mi Casa, Mi Vida o "el plan de las 12.000 viviendas", que era la meta que se iba a alcanzar en la producción de unidades habitacionales.

El desarrollo del programa comprendió el período 2003-2010, y su financiamiento se solventó a través de un crédito otorgado por el Banco Interamericano de Desarrollo (BID), el que abarcó el $83 \%$ del presupuesto. Como contraparte se utilizaron fondos del presupuesto provincial. Su costo fue de US\$ 116.036.200.5

El objetivo general fue contribuir al mejoramiento integral del hábitat y las condiciones de vida de las familias beneficiarias. Específicamente, la intervención de este programa estuvo dirigida a los grupos que habitaban en zonas de riesgo y fueron afectados por las inundaciones del río Suquía, sus afluentes y canales maestros. Las familias fueron relocalizadas en nuevos conjuntos compuestos por unidades habitacionales (viviendas familiares de 42 $\mathrm{m}^{2}$ ) e infraestructura completa (agua, electricidad, alumbrado público, asfalto y cloacas). En el caso de los barrios con más de 250 viviendas, también contaron con equipamiento (escuelas, centro de salud, posta policial) y fueron denominados "barrios-ciudades" (ejemplo, Ciudad Evita, Ciudad de Mis Sueños, Ciudad Sol Naciente, etc.).
5. El costo de cada solución habitacional no debia superar U\$S 8600, con infraestructura de servicios básicos, equipamiento social mínimo y terreno. 
6. Se sostienen en los nueve barrios-ciudades situaciones de pobreza estructural; el porcentaje de hogares con NBI varía entre el 15 al $21 \%$ en esos complejos habitacionales, siendo el indicador predominante el de hacinamiento.

\section{LA DINÁMICA DE LA SRS Y LA INCIDENCIA DE LA POLÍTICA PÚBLICA DE VIVIENDA SOCIAL}

Durante el período 2001-2008 - como mencionamos anteriormente—, se ha desarrollado el programa Mi Casa, Mi Vida. Si bien no tuvo como objetivo abordar el fenómeno de la segregación, su acción generó efectos en la configuración de la ciudad y la localización residencial de población de bajos ingresos, lo cual podemos comprender, en términos de BOURDIEU (1999), como una construcción política del espacio.

Entre los años 2003-2008, por medio de esta operatoria, se ha realizado el traslado de aproximadamente setenta villas, adjudicado 8876 nuevas viviendas emplazadas en 39 nuevos barrios, de los cuales nueve fueron "barrios-ciudades" con más de 250 viviendas. Con un fuerte criterio de focalización de sus destinatarios, la población objetivo de este programa fueron los pobres, específicamente los residentes en villas de emergencia, incapaces de acceder a una vivienda vía el mercado y que necesitan mejores condiciones de habitabilidad, por lo cual la entrega de la vivienda nueva es totalmente subsidiada.

El accionar de este programa ha estado dirigido a disminuir la cantidad de hogares con déficit cuantitativo de viviendas, a través de la implantación de conjuntos habitacionales compuestos por viviendas, equipamiento e infraestructura completa, con un fuerte carácter sectorial, que reproduce la lógica de los anteriores planes de entrega de vivienda llave en mano. En el año 2001, la cantidad de hogares con déficit habitacional cuantitativo en la ciudad de Córdoba era de 20.311 (RODRÍGUEZ Y TABORDA, 2009); la implementación de este programa ha representado una respuesta para el $43,7 \%$ de ellos, con la ejecución de las viviendas.

El acceso a una unidad habitacional y a los servicios públicos ha representado mejores condiciones de vida para muchas familias; sin embargo, no podemos decir que los problemas habitacionales se hayan resuelto. Las dimensiones de la vivienda $\left(42 \mathrm{~m}^{2}\right.$, con dos dormitorios) han sido diseñadas desde parámetros del modelo de familia nuclear, no se reconocen otros tipos de hogares (nuclear numerosa, extensos, etc.), lo que obliga a "acomodarse" a reducidos espacios en cada ambiente, según umbrales críticos que pueden tener como consecuencias problemas psicológicos, afectivos, etc. (ElORZA, 2008); esto ha llevado que se continúen reproduciendo condiciones habitacionales deficitarias. ${ }^{6}$

Los nuevos barrios de vivienda social son concebidos como una ciudad dentro de la ciudad, y el proyecto responde a plasmar el costo de eficiencia en el polígono de intervención, 
mientras que el diseño incorpora una organización espacial propia y diferenciada del entorno (Rodulfo, 2006). Esta planificación se expresa en la ejecución de Mi Casa, Mi Vida, ya que se reproduce una clonación de tipologías de las viviendas y del equipamiento social, los espacios verdes y el ingreso a los barrios-ciudades a través de un arco de entrada, a modo de lo que RodRíguez Chumillas (2006) denomina “cerrada popular". Se explicita la generalización de unos modos de habitar que sugieren una mayor diferenciación en la apropiación del espacio y nuevos paisajes y arquitecturas de la exclusión.

Segregación Residencial Socioeconómica - Localización de barrios de Vivienda Social - Ciudad de Córdoba 2008

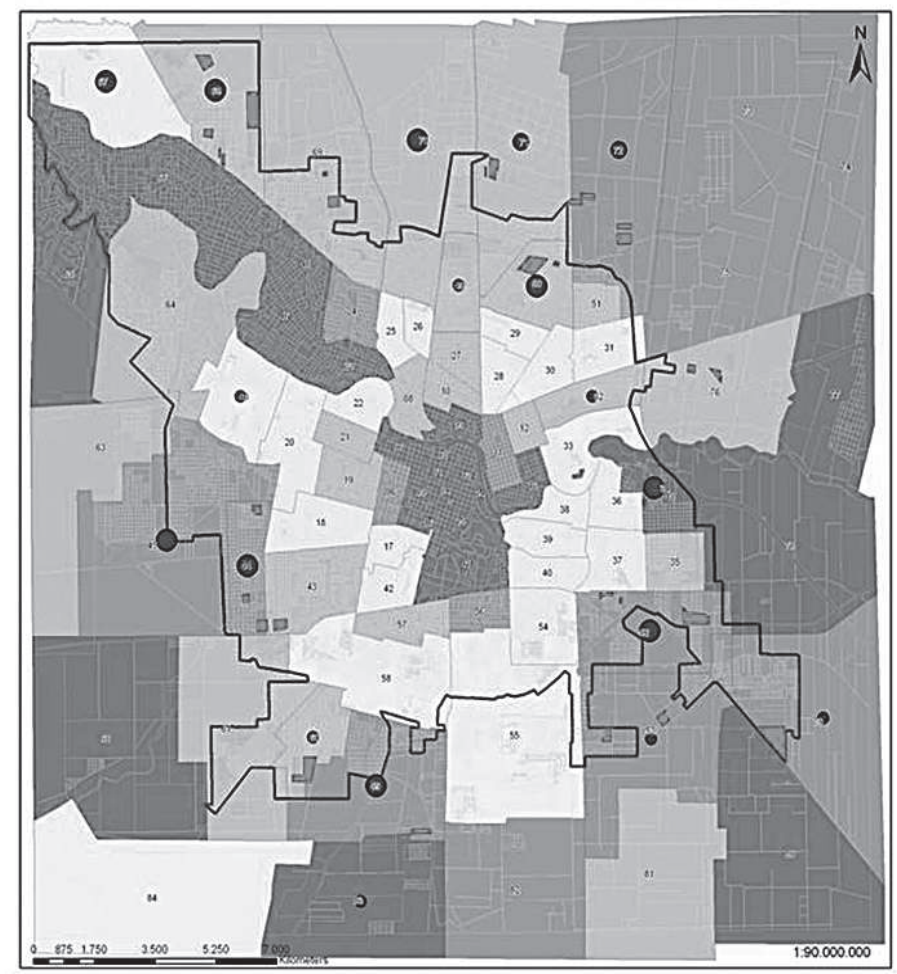

\section{REFERENCIAS}

Desviación Estandar de la media

Variación del 1 al $10 \%$ - Nivel bajo de segregación

Variación del 11 al $20 \%$ (por encima de la media)

Nivel moderado de segregación

Variación del 11 al $20 \%$ (por debajo de la media)

Nivel moderado de segregación

Variación del 21 al $30 \%$ (por encima de la media)

Nivel alto de segregación

Variación del 21 al $30 \%$ (por debajo de la media) Nivel alto de segregación

Nivel alto de segregación

Variación mas del $30 \%$ (por encimación
Nivel muy alto de segregación

Variación mas del $30 \%$ (por debajo de la media)

Nivel muy alto de segregación

Desviacion Estandar de la media NBI

- $1040,00-2000,00$

- $2000,01-3000,00$

$2000,01-3000,00$
$3000,01-6216,00$

Localización de barrios de vivienda social Programa "Mi Casa, Mi Vida"

Fuente: Censo Provincial de Poblacion y Vivienda 2008 
Un aspecto central para el análisis de la segregación es conocer la localización de estos proyectos habitacionales en la ciudad. Un factor determinante en la definición de su localización es el costo del suelo, por lo que estos nuevos barrios en su mayoría se encuentran en áreas periféricas. Los nuevos barrios se localizan en áreas de extensión urbana, que generalmente incorporan suelos que estaban afectados al uso rural, con la consecuente demanda de extensión de infraestructura y servicios públicos y las implicancias en los costos de urbanización $\mathrm{y}$ de funcionamiento, que deben ser asumidos por el municipio.

La localización de los conjuntos habitacionales ha configurado una nueva distribución de la población pobre en la ciudad trasladándolos del anillo central e intermedio a sectores periféricos, en los que los servicios, equipamientos y recursos son de menor calidad. En la figura $\mathrm{N}^{\circ} 3$ se ha superpuesto la espacialización de la SRS y la nueva localización de los barrios ejecutados por Mi Casa, Mi Vida. Se identifica la ubicación de los nuevos complejos de vivienda social en los sectores periféricos, en especial en el sudeste y nordeste de la ciudad, caracterizados por una concentración de población en condiciones de pobreza. De esta manera se contribuye a reforzar la homogeneidad de estos territorios, en cuanto a las características económicas, sociales y culturales de su población.

Asimismo, la conformación de territorios homogéneos en cuanto a la composición social de los grupos responde a los criterios de focalización de las familias destinatarias de este programa, sin haber incorporado criterios de mixtura social. La nueva localización ha disminuido la posibilidad que le daba la antigua residencia de que los vecinos experimentaran interacciones con sujetos de otros grupos sociales, que en muchos casos representaban oportunidades de acceso a fuentes de trabajo (Montoya Flores, 2008; Elorza, 2014). Como afirma SABATINI (2003), cuando la homogeneidad social del espacio es muy fuerte, los efectos tienden a ser negativos, en especial cuando se relacionan con su carácter involuntario. Es decir, cuando los sectores pobres son expulsados de ciertas áreas de la ciudad, sea como consecuencia del mercado de suelo o por intervenciones públicas que los empujan a la periferia.

La erradicación de las familias de las villas ha tenido efectos en la vida cotidiana de la población, ya que han mejorado sus condiciones materiales de vida, pero deben asumir mayores costos de movilidad urbana y tiempos de traslado, adaptación de estrategias de reproducción social, pérdida de oportunidades de inserción laboral, ruptura de redes sociales, etc. (Montoya Flores, 2008; ElORZA, 2008). La revisión de esta política y los 
resultados de su implementación permite inscribir un claro componente de diferenciación social y profundización del desigual acceso a la ciudad y las oportunidades que esta brinda según la condición socioeconómica de los sujetos. Se introduce una serie de interrogantes en relación con la producción y profundización de segregación residencial socioeconómica y la calidad de vida de las familias destinatarias.

La distancia a los espacios de centralidad urbana, la imposibilidad para la población de afrontar gastos de transporte y la calidad deficiente de ese servicio, la baja mixtura social, el deterioro edilicio y la reproducción de condiciones habitacionales deficientes son algunos de los principales factores que contribuyen a la fragmentación urbana y que están configurando nuevos problemas, necesidades y demandas de los habitantes de barrios de vivienda social.

\section{CONCLUSIONES}

En este artículo abordamos la relación entre la SRS y la política pública de vivienda social. La revisión de estudios producidos en la región latinoamericana referidos a este tema da cuenta de los cambios ocurridos en los enfoques de intervención de las políticas sociales sobre la cuestión social habitacional, a partir de las transformaciones ocurridas en las ciudades como consecuencia de los cambios en el régimen de acumulación capitalista y la orientación de las políticas habitacionales facilitadoras y focalizadas de alivio de la pobreza. Con la prevalencia de la lógica del mercado, los problemas asociados con la calidad de vida urbana de la población se han profundizado: la localización de los proyectos habitacionales está cada vez más alejada de los sectores de centralidad, las viviendas tienen superficies mínimas habitables con peor calidad constructiva, conjuntos de gran tamaño, lo que tiene como consecuencia la concentración de población pobre en determinados barrios de la ciudad y mayor estigma por ser residentes de barrios de vivienda social.

En Argentina en general y Córdoba en particular, las orientaciones de las políticas han estado vinculadas con estos determinantes y presentan las mismas limitaciones y problemáticas que las analizadas en la región; prevalece la política sectorial "viviendista" para abordar el déficit habitacional cuantitativo, como estrategia de desarrollo económico y promoción del empleo y también de sostenimiento del subsidio para las empresas constructoras. 
El estudio realizado lleva a corroborar la hipótesis referida a que los proyectos de vivienda social implementados a través de políticas públicas focalizadas han incidido en la intensificación de la segregación residencial y refuerzan las condiciones de reproducción de la pobreza durante el período analizado, ya que la orientación y la intervención de esta política han tenido como impacto una nueva configuración de la ciudad en lo referente a la localización residencial de la población de bajos ingresos, la erradicación de villas y la producción de nuevos territorios de relegación (WACQUANT, 2001).

La erradicación de las villas ha incidido en la profundización de la segregación residencial de la población de altos ingresos, ya que no comparten el espacio con los villeros, lo que posibilitaba cierta mixtura social en esos espacios y representaba posibilidades de mayor acceso a servicios y oportunidades laborales, culturales y sociales para las familias residentes en las villas. Como contracara, la localización de los nuevos barrios en sectores periféricos de la ciudad y la composición social de los grupos destinatarios de este programa (los pobres residentes de las villas) ha sostenido la concentración de población en condiciones de pobreza. Esto ha contribuido a reforzar la homogeneidad de estos territorios, en cuanto a las características económicas, sociales y culturales de su población.

Asimismo, las limitaciones en el diseño de la tipología de la vivienda, que no reconoce la importancia de la conformación de los hogares, ha tenido como consecuencia una reproducción de condiciones habitacionales deficitarias, en las que predominan las situaciones de hacinamiento. Sumado a esto, la denominación de estos conjuntos como "barrios- ciudades", la disposición de su ingreso por medio de arcos que significan una ruptura con el tejido urbano y la localización de equipamientos sociales (dispensario, escuela, comisaria, etc.) a los que accede la población de estos barrios constituyen factores problemáticos para reflexionar sobre la segregación subjetiva y sus vinculaciones con las dimensiones objetivas, aspectos necesarios para abordar en próximos estudios en busca de comprender el fenómeno de la segregación residencial desde una perspectiva que involucre las dimensiones espaciales, sociales y simbólicas.

Vivir en la periferia y en territorios homogéneos socialmente pobres representa ciertos problemas vinculados con la accesibilidad y la carencia de servicios y equipamientos de cierta calidad en sus lugares de residencia, además de los problemas de desintegración social, los cuales representan formas de empobrecimiento o de degradación social vinculadas con las desventajas que conlleva el aislamiento físico, lo que hace que los grupos populares de nuestras ciudades sean aún más pobres (BRAIN Y SABATINI, 2006). 


\section{BIBLIOGRAFÍA}

ARBACI, Sonia (2008). "Hacia la construcción de un discurso sobre la inmigración en las ciudades del sur de Europa. La política urbanística y de vivienda como mecanismos estructurales de marginación étnica residencial". En: ACE: Architecture, City and Environment $=$ Arquitectura, Ciudad y Entorno. Universidad Politécnica de Catalunya, Barcelona.

ARRIAGADA LUCO, C. y RODRÍGUEZ VIGNOLI, J. (2003). "Segregación residencial en áreas metropolitanas de América Latina: magnitud, características, evolución e implicaciones de política". Serie Población y Desarrollo, 47, octubre, 2003, CEPAL.

GOBIERNO DE LA PROVINCIA DE CÓRDOBA (2003). "Proyecto de emergencia para la rehabilitación habitacional de los grupos vulnerables afectados por las inundaciones en la ciudad de Córdoba, programa Mi Casa, Mi Vida".

BOURDIEU, Pierre (2001) Las estructuras sociales de economía. Ed. Manantial, Buenos Aires, Argentina.

BRAIN, I. y SABATINI, F. (2006). "Los precios del suelo en alza carcomen el subsidio habitacional, contribuyendo al deterioro en la calidad y localización de la vivienda social". En: ProUrbana. Instituto de Estudios Urbanos, Santiago de Chile.

BORJA, Jordi (2007). "Revolución y contrarrevolución en la ciudad global: las expectativas frustradas por la globalización de nuestras ciudades". En: EURE (Santiago). Pontificia Universidad Católica de Chile, Santiago de Chile.

CALDEIRA, Teresa (2010). "Democracia y muros: nuevas articulaciones del espacio público". En: Revista Programa en gestión de la ciudad. Universidad Oberta de Catalunya, Barcelona.

DAVIS, Mike (2006) Planeta de Ciudades Miseria. FOCA, Madrid.

DIRECCIÓN GENERAL DE ESTADÍSTICAS Y CENSO DE LA PROVINCIA

DE CÓRDOBA. "Datos Censo Provincial 2008". Gobierno de la Provincia de Córdoba, Córdoba.

DUHAU, Emilio (2013). "La división social del espacio metropolitano. Una propuesta de análisis". [En línea] Nueva Sociedad. 243: 79-91, enero-febrero 2013. [10 de octubre 2014] ISSN: 0251-3552. Disponible en: http://nuso.org/media/articles/downloads/3917_1.pdf.

ELORZA, Āna Laura (2008) "Estudio comparativo entre un programa de erradicación y otro de mejoramiento barrial dirigidos a población vulnerable de la ciudad de Córdoba". Tesis de Maestría. Gestión y Desarrollo Habitacional, Facultad de Arquitectura, Urbanismo y Diseño de la Universidad Nacional de Córdoba, Argentina. 
(2014) "Segregación residencial en la ciudad de Córdoba. Dinámica y efectos en la calidad de vida de la población pobre segregada". Tesis doctoral. Doctorado en Ciencias Sociales, Facultad de Ciencias Políticas y Sociales de la Universidad Nacional de Cuyo, Mendoza.

ESPINA PRIETO, Mayra (2008) Políticas de atención a la pobreza y la desigualdad. Examinando el rol del Estado en la experiencia cubana. CLACSO, Buenos Aires.

ESQUIVEL, M. T.; MAYA, E.; CERVANTES, J. (2005). "La promoción privada y los grandes conjuntos habitacionales: nuevas modalidades de acceso a la vivienda". En: Scripta Nova. Universidad de Barcelona, España.

HARVEY, David (2000). “Mundos Urbanos Posibles”. En: RAMOS, Ángel Martín (Ed.), Lo Urbano. Ediciones Universidad Politécnica de Catalunya, Barcelona, España.

HIDALGO DATTWYLER, Ricardo (2007). “'Se acabó el suelo en la gran ciudad? Las nuevas periferias metropolitanas de la vivienda social en Santiago de Chile". En: Eure. Pontificia Universidad Católica de Chile, Santiago de Chile.

JARAMILLO, Samuel (1999). "El papel del mercado del suelo en la configuración de algunos rasgos socioespaciales de las ciudades latinoamericanas". En: Territorios. Universidad del Rosario, Bogotá.

MARENGO, Cecilia (2004). "Una aproximación a la segregación residencial, como punto de partida en la formulación de políticas". En: INVI. Universidad de Chile, Santiago de Chile.

MARENGO, C. y ELORZA, A. (2009). “Globalización y Políticas urbanas. La política habitacional focalizada como estrategia para atenuar condiciones de pobreza urbana: los programas implementados en Córdoba y los desafíos pendientes". En: Cuaderno Urbano. Ed. Nobuko / Eudene, Resistencia, Chaco, Argentina.

(2014). "Tendencias de segregación residencial socioeconómica: el caso de Córdoba (Argentina) en el período 2001-2008”. En: EURE. Pontificia Universidad Católica de Chile, Santiago de Chile.

MASSEY, D. y DENTON, N. (1988). “The Dimensions of Residential Segregation". En: Social Forces. Departamento de Sociología de la Universidad de Carolina del Norte, Estados Unidos.

MONTOYA FLOREZ, Olga (2006). "Calidad de vida en un barrio de habitantes erradicados en la periferia de Córdoba. Estudio de caso, barrio Ciudad de mis Sueños. Lineamientos para la gestión ambiental". Tesis de Maestría. Gestión Ambiental. FAUD, UNC, Córdoba. PRADILLA COBOS, Emilio (1987) Capital, Estado y Vivienda en América Latina. Ed. Fontamara, México. 
PROGRAMA DE NACIONES UNIDAS PARA EL DESARROLLO (2009) Segregación Residencial en Argentina. PNUD, Buenos Aires.

RODULFO, Beatriz (2006). "Políticas Habitacionales. Alternativas de intervención y gestión". Ponencia presentada en Seminario Iberoamericano Ciencia y tecnología para el Hábitat Popular. Construcción y participación del conocimiento. Organizado por CEVE y Universidad Católica de Córdoba, realizado los días 1 y 2 de diciembre en la ciudad de Córdoba, Argentina.

RODRÍGUEZ, A. y SUGRANYES, A. (2005) Los con techo. Un desafio para la política de la vivienda social. Ediciones Sur, Santiago de Chile.

RODRÍGUEZ, M. y TABORDA, A. (2009) Análisis de Politicas Públicas. Formación, estilos de gestión y desempeño: políticas de vivienda. Córdoba 1991- 2007. Brujas, Córdoba, Argentina.

RODRÍGUEZ CHUMILLA, Inés (2006). "Vivienda social latinoamericana: la clonación del paisaje de la exclusión". En: ACE: Architecture, City and Environment = Arquitectura, Ciudad y Entorno. Universidad Politécnica de Catalunya, Barcelona.

RODRÍGUEZ VIGNOLI, Jorge (2001) Segregación residencial socioeconómica: ¿qué es?, ¿Cómo se mide?, ¿qué está pasando?, ¿importa? CEPAL/ ECLAC, Santiago, Chile. SABATINI, Francisco (2003) La segregación social del espacio en las ciudades de América Latina. Banco Interamericano de Desarrollo Departamento de Desarrollo Sostenible, Chile.

(2004). "Medición de la segregación residencial: reflexiones metodológicas desde la ciudad latinoamericana". En: Cáceres y Sabatini (Ed.), Barrios Cerrados en Santiago de Chile: entre la exclusión y la integración residencia. Lincoln Institute o Land Policy e Instituto de Geografía, Santiago, Chile.

SABATINI, F., CÁCERES, G. y CERDA, J. (2001). "Segregación residencial en las principales ciudades chilenas: Tendencias de las tres últimas décadas y posibles cursos de acción". En: EURE (Santiago). Pontificia Universidad Católica de Chile, Santiago de Chile. SARAVI, Gonzalo (2008). "Mundos aislados: segregación urbana y desigualdad en la ciudad de México". En: Eure. Pontificia Universidad Católica de Chile, Santiago de Chile. TECCO, C. y FERNÁNDEZ, S. (2005). "Políticas públicas y segregación residencial socio económica en la ciudad de Córdoba, Argentina". VII Seminario Nacional de la Red de Centros Académicos para el estudio de gobiernos locales. Buenos Aires, 15 y 16 de septiembre de 2005. [25 de septiembre de 2015] Disponible en: http://biblioteca.municipios.unq.edu.ar/modules/mislibros/archivos/polpubysegregacionresidencial2005teccofernandez.pdf. 
WACQUANT, Loic (2001) Parias Urbanos. Marginalidad en la ciudad a comienzos de siglo. Ed. Manantial, Buenos Aires, Argentina.

(2007) Los condenados de la ciudad. Gueto, periferias y Estado. Siglo XXI Editores, Buenos Aires, Argentina.

ZILOCCHI, Gustavo (2007) Villas miseria. La vivienda de los más pobres en la etapa industrial moderna 1930-1970. El Cid Editor, Buenos Aires, Argentina. 